\title{
RELAXED SPANNERS FOR DIRECTED DISK GRAPHS
}

\author{
DAVID PELEG ${ }^{1}$ AND LIAM RODITTY ${ }^{2}$ \\ ${ }^{1}$ Department of Computer Science and Applied Mathematics, \\ The Weizmann Institute of Science, Rehovot 76100, Israel \\ E-mail address: david.peleg@weizmann.ac.il \\ 2 Department of Computer Science, Bar-Ilan University, \\ Ramat-Gan 52900, Israel \\ E-mail address: liamr@macs.biu.ac.il
}

\begin{abstract}
Let $(V, \delta)$ be a finite metric space, where $V$ is a set of $n$ points and $\delta$ is a distance function defined for these points. Assume that $(V, \delta)$ has a constant doubling dimension $d$ and assume that each point $p \in V$ has a disk of radius $r(p)$ around it. The disk graph that corresponds to $V$ and $r(\cdot)$ is a directed graph $I(V, E, r)$, whose vertices are the points of $V$ and whose edge set includes a directed edge from $p$ to $q$ if $\delta(p, q) \leq r(p)$. In [8] we presented an algorithm for constructing a $(1+\epsilon)$-spanner of size $O\left(n / \epsilon^{d} \log M\right)$, where $M$ is the maximal radius $r(p)$. The current paper presents two results. The first shows that the spanner of [8] is essentially optimal, i.e., for metrics of constant doubling dimension it is not possible to guarantee a spanner whose size is independent of $M$. The second result shows that by slightly relaxing the requirements and allowing a small perturbation of the radius assignment, considerably better spanners can be constructed. In particular, we show that if it is allowed to use edges of the disk graph $I\left(V, E, r_{1+\epsilon}\right)$, where $r_{1+\epsilon}(p)=(1+\epsilon) \cdot r(p)$ for every $p \in V$, then it is possible to get a $(1+\epsilon)$-spanner of size $O\left(n / \epsilon^{d}\right)$ for $I(V, E, r)$. Our algorithm is simple and can be implemented efficiently.
\end{abstract}

\section{Introduction}

This paper concerns efficient constructions of spanners for disk graphs, an important family of directed graphs. A spanner is essentially a skeleton of the graph, namely, a sparse spanning subgraph that faithfully represents distances. Formally, a subgraph $H$ of a graph $G$ is a $t$-spanner of $G$ if $\delta_{H}(u, v) \leq t \cdot \delta_{G}(u, v)$ for every two nodes $u$ and $v$, where $\delta_{G^{\prime}}(u, v)$ denotes the distance between $u$ and $v$ in $G^{\prime}$. We refer to $t$ as the stretch factor of the spanner. Graph spanners have received considerable attention over the last two decades, and were used implicitly or explicitly as key ingredients of various distributed applications. It is known how to efficiently construct a $(2 k-1)$-spanner of size $O\left(n^{1+1 / k}\right)$ for every weighted undirected graph, and this size-stretch tradeoff is conjectured to be tight. Baswana and

1998 ACM Subject Classification: F.2 ANALYSIS OF ALGORITHMS AND PROBLEM COMPLEXITY, F.2.0 General .

Key words and phrases: Spanners, Directed graphs.

Thanks: Supported in part by grants from the Minerva Foundation and the Israel Ministry of Science.

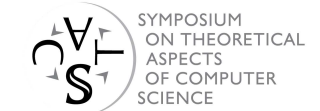

C) D. Peleg and L. Roditty

27th Symposium on Theoretical Aspects of Computer Science, Nancy, 2010

(C) Creative Commons Attribution-NoDerivs License

Editors: Jean-Yves Marion, Thomas Schwentick

Leibniz International Proceedings in Informatics (LIPIcs), Schloss Dagstuhl - Leibniz-Zentrum für Informatik, Germany

Digital Object Identifier: 10.4230/LIPIcs.STACS.2010.2489 
Sen [?] presented a linear time randomized algorithm for computing such a spanner. In directed graphs, however, the situation is different. No such general size-stretch tradeoff can exist, as indicated by considering the example of a directed bipartite graph $G$ in which all the edges are directed from one side to the other; clearly, the only spanner of $G$ is $G$ itself, as any spanner for $G$ must contain every edge.

The main difference between undirected and directed graphs is that in undirected graphs the distances are symmetric, that is, a path of a certain length from $u$ to $v$ can be used also from $v$ to $u$. In directed graphs, however, the existence of a path from $u$ to $v$ does not imply anything on the distance in the opposite direction from $v$ to $u$. Hence, in order to obtain a spanner for a directed graph one must impose some restriction either on the graph or on its distances. In order to bypass the problem of asymmetric distances of directed graphs, Cowen and Wagner [5] introduced the notion of roundtrip distances in which the distance between $u$ and $v$ is composed of the shortest path from $u$ to $v$ plus the shortest path from $v$ to $u$. It is easy to see that under this definition distances are symmetric also in directed graphs. It is shown by Cowen and Wagner [5] and later by Roditty, Thorup and Zwick [6] that methods of path approximations from undirected graphs can work using more ideas also in directed graphs when roundtrip distances are considered. Bollobás, Coppersmith and Elkin [?] introduced the notion of distance preservers and showed that they exist also in directed graphs.

In [8] we presented a spanner construction for directed graphs without symmetric distances. The restriction that we imposed on the graph was that it must be a disk graph. More formally, let $(V, \delta)$ be a finite metric space of constant doubling dimension $d$, where $V$ is a set of $n$ points and $\delta$ is a distance function defined for these points. A metric is said to be of constant doubling dimension if a ball with radius $r$ can be covered by at most a constant number of balls of radius $r / 2$. Every point $p \in V$ is assigned with a radius $r(p)$. The disk graph that corresponds to $V$ and $r(\cdot)$ is a directed graph $I(V, E, r)$, whose vertices are the points of $V$ and whose edge set includes a directed edge from $p$ to $q$ if $q$ is inside the disk of $p$, that is, $\delta(p, q) \leq r(p)$. In [8] we presented an algorithm for constructing a $(1+\epsilon)$-spanner with size $O\left(n / \epsilon^{d} \log M\right)$, where $M$ is the maximal radius. In the case that we remove the radius restriction the resulted graph is the complete undirected graph where the weight of every edge is the distance between its endpoint. In such a case it is possible to create $(1+\epsilon)$-spanners of size $O\left(n / \epsilon^{d}\right)$, see [4], [2] and [9] for more details. Moreover, when the radii are all the same and the graph is the unit disk graph then it is also possible to create $(1+\epsilon)$-spanners of size $O\left(n / \epsilon^{d}\right)$, see [3], [8].

As a result of that, a natural question is whether a spanner size of $O\left(n / \epsilon^{d} \log M\right)$ in the case of directed disk graph is indeed the best possible or maybe it is possible to get a spanner of size $O\left(n / \epsilon^{d}\right)$ as in the cases of the complete graph and the unit disk graph. For the case of the Euclidean metric space, the answer turns out to be positive; a simple modification of the Yao graph construction [11] to fit the directed case yields a directed spanner of size $O\left(n / \epsilon^{d}\right)$. However, the question remains for more general metric spaces, and in particular for the important family of metric spaces of bounded doubling dimension.

In this paper we provide an answer for this question. We show that our construction from [8] is essentially optimal by providing a metric space with a constant doubling dimension and a radius assignment whose corresponding disk graph has $\Omega\left(n^{2}\right)$ edges and none of its edges can be removed. (This does not contradict our spanner construction from [8] as the maximal radius in that case is $\Theta\left(2^{n}\right)$ and hence $\log M=n$.) 
This (essentially negative) optimality result motivates our main interest in the current paper, which focuses on attempts to slightly relax the assumptions of the model, in order to obtain sparser spanner constructions. Indeed, it turns out that such sparser spanner constructions are feasible under a suitably relaxed model. Specifically, we demonstrate the fact that if a small perturbation of the radius assignment is allowed, then a $(1+\epsilon)$-spanner of size $O\left(n / \epsilon^{d}\right)$ is attainable. More formally, we show that if we are allowed to use edges of the disk graph $I\left(V, E, r_{1+\epsilon}\right)$, where $r_{1+\epsilon}(p)=(1+\epsilon) \cdot r(p)$ for every $p \in V$, then it is possible to get a $(1+\epsilon)$-spanner of size $O\left(n / \epsilon^{d}\right)$ for the original disk graph $I(V, E, r)$. This approach is similar in its nature to the notation of emulators introduced by Dor, Halperin and Zwick [1]. An emulator of a graph may use any edge that does not exist in the graph in order to approximate its distances. It was used in the context of spanners with an additive stretch.

The main application of disk graph spanners is for topology control in the wireless ad hoc network model. In this model the power required for transmitting from $p$ to $q$ is commonly taken to be $\delta(p, q)^{\alpha}$, where $\delta(p, q)$ denotes the distance between $p$ and $q$ and $\alpha$ is a constant typically assumed to be between 2 and 4 . Most of the ad hoc network literature makes the assumption that the transmission range of all nodes is identical, and consequently represents the network by a unit disk graph (UDG), namely, a graph in which two nodes $p, q$ are adjacent if their distance satisfies $\delta(p, q) \leq 1$. A unit disk graph can have as many as $O\left(n^{2}\right)$ edges.

There is an extensive body of literature on spanners of unit disk graphs. Gao et al. [3], Wang and Yang-Li [10] and Yang-Li et al. [7] considered the restricted Delaunay graph, whose worst-case stretch is constant (larger than $1+\epsilon)$. In [8] we showed that any $(1+\epsilon)$ geometric spanner can be turned into a $(1+\epsilon)$-UDG spanner.

Disk graphs are a natural generalization of unit disk graphs, that provide an intermediate model between the complete graph and the unit disk graph. Our size efficient spanner construction for disk graphs whose radii are allowed to be slightly larger falls exactly into the model of networks in which the stations can change their transmission power. In particular our constriction implies that if any station increases its transmission power by a small fraction then a considerably improved topology can be built for the network.

Our result has both practical and theoretical implications. From a practical point of view it shows that, in certain scenarios, extending the transmission radii even by a small factor can significantly improve the overall quality of the network topology. The result is also very intriguing from a theoretical standpoint, as to the best of our knowledge, our relaxed spanner is the first example of a spanner construction for directed graphs that enjoys the same properties as the best constructions for undirected graphs. (As mentioned above, it is easy to see that for general directed graphs, it is not possible to have an algorithm that given any directed graph produces a sparse spanner for it.) In that sense, our result can be viewed as a significant step towards gaining a better understanding for some of the fundamental differences between directed and undirected graphs. Our result also opens several new research directions in the relaxed model of disk graphs. The most obvious research questions that arise are whether it is possible to obtain other objects that are known to exist in undirected graphs, such as compact routing schemes and distance oracles, for disk graphs as well.

The rest of this paper is organized as follows. In the next section we present a metric space of constant doubling dimension in which no edge can be removed from its corresponding disk graph. Section 2 first describes a simple variant of our construction from [8], 


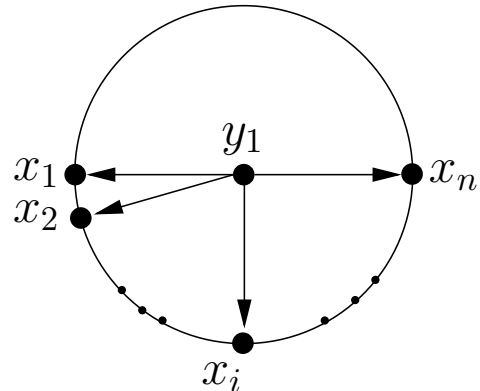

(a)

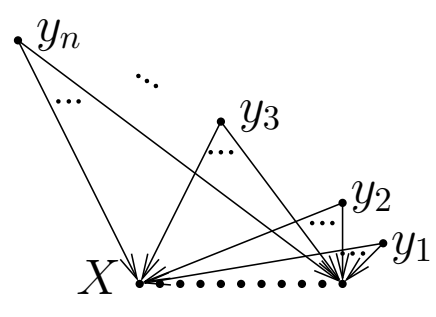

(b)

Figure 1: (a) First step in constructing the non-sparsifiable disk graph G. (b) The nonsparsifiable disk graph $G$.

and then uses it together with new ideas in order to obtain our new relaxed construction. Finally, in Section 3 we present some concluding remarks and open problems.

\section{Optimality of the spanner construction}

In this section we build a disk graph $G$ with $2 n$ vertices and $\Omega\left(n^{2}\right)$ edges that is nonsparsifiable, namely, whose only spanner is $G$ itself. In this graph $M=\Omega\left(2^{n}\right)$ hence our spanner construction from [8] has a size of $\Omega\left(n^{2}\right)$ and is essentially optimal.

Given a set of points, we present a distance function such that for a given assignment of radii for the points any spanner of the resulting disk graph must have $\Omega\left(n^{2}\right)$ edges. We then prove that the underlying metric space has a constant doubling dimension.

We partition the points into two types, $Y=\left\{y_{1}, \ldots, y_{n}\right\}$ and $X=\left\{x_{1}, \ldots, x_{n}\right\}$. We now define the distance function $\delta(\cdot, \cdot)$ and the radii assignment $r(\cdot)$. The main idea is to create a bipartite graph $G(X, Y, E)$ in which every point of $Y$ is connected by a directed edge to all the points of $X$.

The distance between any two points $x_{i}$ and $x_{j}$ is at least $1+\epsilon$ for some small $0<\epsilon<1$ and the radius assignment of every point $x_{i}$ is exactly 1 . Thus, there are no edges between the points of $X$.

We now define the distances between the points of $Y$ and the points of $X$. We start with the point $y_{1}$. Let $\delta\left(y_{1}, x_{i}\right)=n$ for every $x_{i} \in X$ and let $r\left(y_{1}\right)=n$. Place the points of $X$ on the boundary of a ball of radius $n$ centered at $y_{1}$ such that the distance between any two consecutive points $x_{i}$ and $x_{i+1}$ is exactly $1+\epsilon$. This is depicted in Figure 1(a).

Turning to the point $y_{2}$, let $\delta\left(y_{2}, x_{i}\right)=2 n$ for every $x_{i} \in X, \delta\left(y_{2}, y_{1}\right)=2 n+\epsilon$, and $r\left(y_{2}\right)=2 n$. Hence there is an edge from $y_{2}$ to all the points of $X$, but no edge connects $y_{2}$ and $y_{1}$.

We now turn to define the general case. Consider $y_{i} \in Y$. Let $r\left(y_{i}\right)=2^{i-1} n$ and $\delta\left(y_{i}, x_{j}\right)=2^{i-1} n$ for every $x_{j} \in X$. Let $\delta\left(y_{i}, y_{i-1}\right)=2^{i-1} n+\epsilon$, and in general, for every $0<j<i$ we have

$$
\delta\left(y_{i}, y_{j}\right)=\sum_{k=j}^{i-1} \delta\left(y_{k+1}, y_{k}\right)
$$


implying that

$$
\delta\left(y_{i}, y_{j}\right)<2^{i} n .
$$

It is easy to verify that $y_{i}$ has outgoing edges to the points of $X$ (and to them only) and it does not have any incoming edges. See Figure 1(b).

The resulting disk graph $G$ has $2 n$ vertices and $\Omega\left(n^{2}\right)$ edges. Clearly, removing any edge from $G$ will increase the distance between its head and its tail to infinity, and thus the only spanner of $G$ is $G$ itself.

It is left to show that the metric space defined above for $G$ has a constant doubling dimension. Given a metric space $(V, \delta)$, its doubling dimension is defined to be the minimal value $d$ such that every ball $B$ of radius $r$ in the metric space can be covered by $2^{d}$ balls of radius $r / 2$. In the next Theorem we prove that for the metric space described above, $d$ is constant.

Theorem 1.1. The metric space $(X \cup Y, \delta)$ defined for $G$ has a constant doubling dimension.

Proof. Let $B$ be a ball with an arbitrary radius $r$. We show that it is possible to cover all the points of $X \cup Y$ within $B$ using a constant number of balls whose radius is $r / 2$. The proof is divided into two cases.

Case a: There is some $y_{j} \in Y$ within the ball $B$. (If there is more than one such point, then let $y_{j}$ be the point whose index is maximal.) Let $B^{\prime}$ be a ball of radius $R=2 r$ centered at $y_{j}$. Clearly $B \subset B^{\prime}$, so $B^{\prime}$ contains all the points of $B$. In what follows we show that all the points of $X \cup Y$ within $B^{\prime}$ can be covered by a constant number of balls of radius $r / 2$. Let $y_{i}$ be the point within $B^{\prime}$ whose index is maximal. We have to consider two possible scenarios. The first is that $y_{j}=y_{i}$. This implies that $y_{j+1} \notin B^{\prime}$, hence $R<\delta\left(y_{j+1}, y_{j}\right)=2^{j} n+\epsilon$. We now show that it is possible to cover $B^{\prime}$ by a constant number of balls of radius $R / 4$. If $R<2^{j-1} n$, then only $y_{j}$ is within $B^{\prime}$ and it is covered by a ball of radius $R / 4$ centered at itself. If $2^{j-1} n \leq R<2^{j-1} n+\epsilon$, then $B^{\prime}$ contains all the points of $X$ and $y_{j}$. From packing arguments it follows that it is possible to cover all the points of $X$ by a constant number of balls of radius $n / 4$, hence also by a constant number of balls of radius $R \geq n$. The point $y_{j}$ itself is covered by a ball centered at it. Finally, if $2^{j-1} n+\epsilon \leq R<2^{j} n+\epsilon$, then $R / 4$ is at least $2^{j-3} n+\epsilon / 4$. A ball centered at $y_{j-3}$ of radius $R / 4$ covers every $y_{k}$ within $B^{\prime}$, where $1 \leq k \leq j-3$, as $\delta\left(y_{j-3}, y_{k}\right) \leq 2^{j-3} n$. Hence, we cover $Y \cap B^{\prime}$ by balls of radius $R / 4$ whose centers are $y_{j}, y_{j-1}, y_{j-2}$ and $y_{j-3}$. We cover $X \cap B^{\prime}$ as before. This completes the first scenario, where $y_{i}=y_{j}$. Assume now that $y_{i} \neq y_{j}$. This implies that $\delta\left(y_{i}, y_{j}\right) \leq R$ and that $R<\delta\left(y_{i+1}, y_{j}\right)$, where the first inequality follows from the fact that $y_{i} \in B^{\prime}$ and the second inequality follows from the fact that $y_{i}$ is the point with maximal index inside $B^{\prime}$, hence, $y_{i+1} \notin B^{\prime}$. As $\delta\left(y_{i}, y_{i-1}\right) \leq \delta\left(y_{i}, y_{j}\right)$, we get that $2^{i-1} n+\epsilon \leq R$. Also, by (1.2), $\delta\left(y_{i+1}, y_{j}\right)<2^{i+1} n$. We conclude that $2^{i-1} n \leq R<2^{i+1} n$ and that $R / 4 \geq 2^{i-3} n$. A ball centered at $y_{i-3}$ of radius $R / 4$ covers every $y_{k}$ within $B^{\prime}$, where $k \leq i-3$, as $\delta\left(y_{i-3}, y_{k}\right) \leq 2^{i-3} n$. Hence, we can cover $B^{\prime} \cap Y$ by balls of radius $R / 4$ whose centers are $y_{i}, y_{i-1}, y_{i-2}$ and $y_{i-3}$. We cover $X \cap B^{\prime}$ as before. This completes the first case.

Case b: The ball $B$ does not contain any point from $Y$. The points of $X$ are spread as appears in Figure 1(a), thus by standard packing arguments, any ball that contains only points from $X$ is covered by a constant number of balls of half the radius. 


\section{Improved spanner in the relaxed disk graph model}

The (negative) optimality result from the previous section motivates us to look for a slightly relaxed definition of disk graphs in which it will still be possible to create a spanner of size $O\left(n / \epsilon^{d}\right)$.

Let $(V, \delta)$ be a metric space of constant doubling dimension $d$ with a radius assignment $r(\cdot)$ for its points and let $I=(V, E, r)$ be its corresponding disk graph. Assume that we multiply the radius assignment of every point by a factor of $1+\epsilon$, for some $\epsilon>0$, and let $I^{\prime}=\left(V, E^{\prime}, r_{1+\epsilon}\right)$ be the corresponding disk graph. It is easy to see that $E \subseteq E^{\prime}$. In this section we show that it is possible to create a $(1+\epsilon)$-spanner of size $O\left(n / \epsilon^{d}\right)$ if we are allowed to use edges of $I^{\prime}$. As a first step we present a simple variant of our $(1+\epsilon)$-spanner construction of size $O\left(n / \epsilon^{d} \log M\right)$ from [8]. This variation is needed in order to obtain the efficient construction in the relaxed model which is presented right afterwards.

\subsection{Spanners for general disk graphs}

Let $(V, \delta)$ be a metric space of constant doubling dimension and assume that any point $p \in V$ is the center of a ball of radius $r(p)$, where $r(p)$ is taken from the range $[1, M]$. In this section we describe a simple variant of our construction from [8], which computes a $(1+\epsilon)$-spanner with $O\left(n / \epsilon^{d} \log M\right)$ edges for a given disk graph. We then use this variant, together with new ideas, in order to obtain (in the next section) our main result, namely, a spanner with only $O\left(n / \epsilon^{d}\right)$ edges.

The spanner construction algorithm receives as input a directed graph $I(V, E, r)$ and an arbitrarily small (constant) approximation factor $\epsilon>0$, and constructs a set of spanner edges $E_{\mathrm{SP}}^{\mathrm{DIR}}$, returning the spanner subgraph $H^{\mathrm{DIR}}\left(V, E_{\mathrm{SP}}^{\mathrm{DIR}}\right)$. The construction of the spanner is based on a hierarchical partition of the points of $V$ that takes into account the different radius of each point. The construction operates as follows. Let $\alpha$ and $\beta$ be two small constants depending on $\epsilon$, to be fixed later on. Assume that the ball radii are scaled so that the smallest edge in the disk graph is of weight 1 . Let $i$ be an integer from the range $\left[0,\left\lfloor\log _{1+\alpha} M\right\rfloor\right]$ and let $M_{i}=M /(1+\alpha)^{i}$. The edges of $I(V, E, r)$ are partitioned into classes by length, letting $E\left(M_{i+1}, M_{i}\right)=\left\{(x, y) \mid M_{i+1} \leq \delta(x, y) \leq M_{i}\right\}$. Let $\ell(x, y)$ be the level of the edge $(x, y)$, that is, $\ell(x, y)=i$ such that $(x, y) \in E\left(M_{i+1}, M_{i}\right)$. Let $p$ be a point whose ball is of radius $r(p) \in\left[M_{i+1}, M_{i}\right]$. It follows that level $i$ is the first level in which $p$ can have outgoing edges. We denote this level by $\ell(p)$.

For every $i \in\left[0,\left\lfloor\log _{1+\alpha} M\right\rfloor\right]$, starting from $i=0$, the edges of the class $E\left(M_{i+1}, M_{i}\right)$ are considered by the algorithm in a non-decreasing order. (Assume that in each class the edges are sorted by their weight.) In each stage of the construction we maintain a set of pivots $P_{i}$. Let $x \in V$ and let $\mathrm{NN}\left(x, P_{i}\right)$ be the nearest neighbor of $x$ among the points of $P_{i}$. For a pivot $p \in P_{i}$, define $\Gamma_{i}(p)=\left\{x \mid x \in V, \mathrm{NN}\left(x, P_{i}\right)=p, r(x) \geq \delta(x, p)\right\}$, namely, all the points that have a directed edge to $p$ and $p$ is their nearest neighbor from $P_{i}$. We refer to $\Gamma_{i}(p)$ as the close neighborhood of $p$.

The algorithm is given in Figure 2. Let $(x, y)$ be an edge considered by the algorithm in the $i$ th iteration. The algorithm first checks whether $x$ or $y$ or both should be added to the pivots set $P_{i}$. The main change with respect to [8] is that if $y$ is assigned with a large enough radius it might become a pivot when the edge $(x, y)$ is examined. When considering the edge $(x, y)$, the algorithm acts according to the following rule: If the distance from $x$ to its nearest neighbor in $P_{i}$ is greater than $\beta M_{i+1}$ then $x$ is added to $P_{i}$. If the distance from $y$ to its nearest neighbor in $P_{i}$ is greater than $\beta M_{i+1}$ and the radius of $y$ 


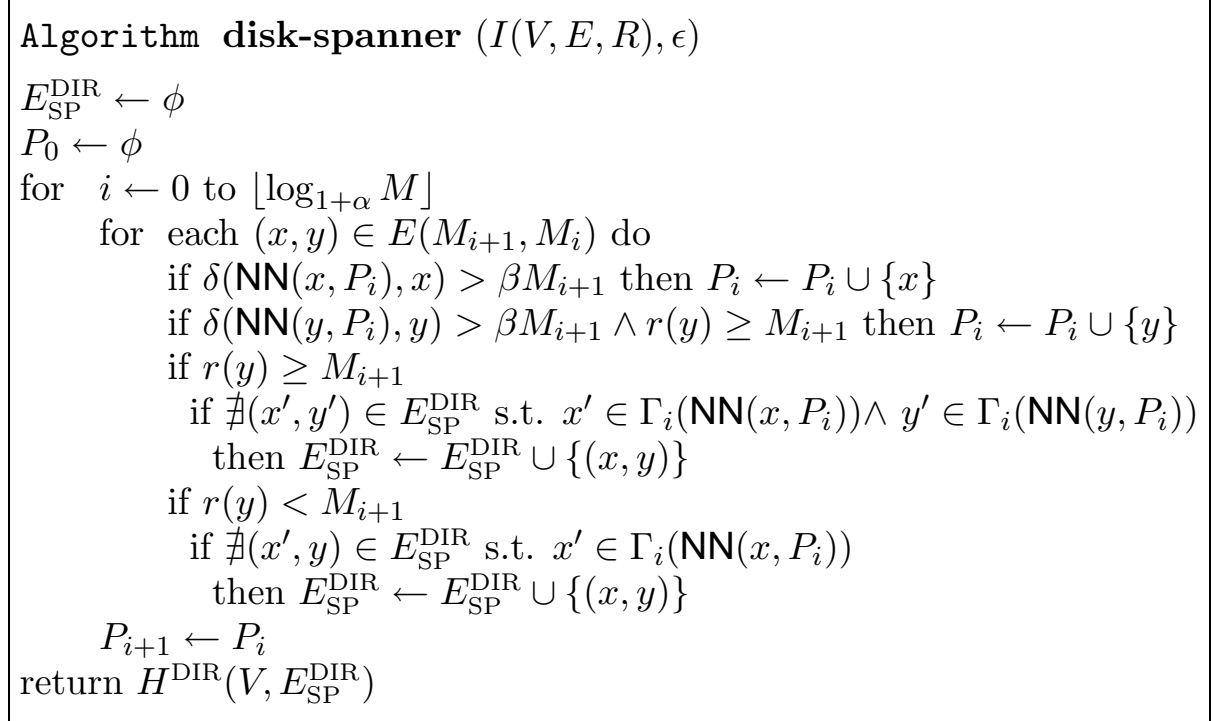

Figure 2: A high level implementation of the spanner construction algorithm for general disk graphs

is at least $M_{i+1}$ then $y$ is added to $P_{i}$. To decide whether the edge $(x, y)$ is added to the spanner, the following two cases are considered. The first case is when $r(y) \geq M_{i+1}$. In this case, if there is no edge from the close neighborhood of $x$ to the close neighborhood of $y$ then $(x, y)$ is added to the spanner. The second case is when $r(y)<M_{i+1}$. In this case, if there is no edge from the close neighborhood of $x$ to $y$ then $(x, y)$ is added to the spanner. When $i$ reaches $\left\lfloor\log _{1+\alpha} M\right\rfloor$, the algorithm handles all the edges that belong to $E\left(M_{\left\lfloor\log _{1+\alpha} M\right\rfloor+1}, M_{\left\lfloor\log _{1+\alpha} M\right\rfloor}\right)$. This includes also edges whose weight is 1 , the minimal possible weight. The algorithm returns the directed graph $H^{\mathrm{DIR}}\left(V, E_{\mathrm{SP}}^{\mathrm{DIR}}\right)$.

In what follows we prove that for suitably chosen $\alpha$ and $\beta, H^{\mathrm{DIR}}\left(V, E_{\mathrm{SP}}^{\mathrm{DIR}}\right)$ is a $(1+\epsilon)$ spanner with $O\left(n / \epsilon^{d} \log M\right)$ edges of the directed graph $I(V, E, r)$.

Lemma 2.1 (Stretch). Let $\epsilon>0$, set $\alpha=\beta<\epsilon / 6$ and let $H=H^{D I R}\left(V, E_{S P}^{D I R}\right)$ be the graph returned by Algorithm disk-spanner $(I(V, E, r), \epsilon)$. If $(x, y) \in E$ then $\delta_{H}(x, y) \leq$ $(1+\epsilon) \delta(x, y)$.

Proof. Recall that the radii are scaled so that the shortest edge is of weight 1 . We prove that every directed edge of an arbitrary node $x \in V$ is approximated with $1+\epsilon$ stretch. Let $i \in\left[0,\left\lfloor\log _{1+\alpha} M\right\rfloor\right]$. The proof is by induction on $i$. For a given node $x$, the base of the induction is the maximal value of $i$ in which $x$ has an edge in $E\left(M_{i+1}, M_{i}\right)$. Let $j$ be this value for $x$, that is, the set $E\left(M_{j+1}, M_{j}\right)$ contains the shortest edge that touches $x$. Every other node is at distance at least $M_{j+1}$ away from $x$, hence $x$ is a pivot at this stage and every edge that touches $x$ from the set $E\left(M_{j+1}, M_{j}\right)$ is added to $E_{\mathrm{SP}}^{\mathrm{DIR}}$.

Let $(x, y) \in E\left(M_{i+1}, M_{i}\right)$ for some $i<j$ and let $p=\mathrm{NN}\left(x, P_{i}\right)$. Assume that $r(y) \geq$ $M_{i+1}$ and let $q=\mathrm{NN}\left(y, P_{i}\right)$. It follows from definition that $\delta(x, p) \leq \beta M_{i+1}$ and $\delta(y, q) \leq$ $\beta M_{i+1}$.

If the edge $(x, y)$ is not in the spanner, then there must be an edge $(\hat{x}, \hat{y}) \in E_{\mathrm{SP}}^{\mathrm{DIR}}$, where $\hat{x} \in \Gamma_{i}(p)$ and $\hat{y} \in \Gamma_{i}(q)$. The crucial observation is that the radius of $x$ and $\hat{y}$ is at least $M_{i+1}$. By the choice of $\beta$, it follows that $2 \beta M_{i+1}<M_{i+1}$ and $(x, \hat{x}),(\hat{y}, y) \in E$. Thus, 
there is a (directed) path from $x$ to $y$ of the form $\langle x, \hat{x}, \hat{y}, y\rangle$ whose length is $4 \beta M_{i+1}+M_{i}$. However, only its middle edge, $(\hat{x}, \hat{y})$, is in $E_{\mathrm{SP}}^{\mathrm{DIR}}$. The length of this edge is bounded by the length of the edge $(x, y)$ since the algorithm picked the minimal edge that connects between the neighborhoods. This implies that the length of $(\hat{x}, \hat{y})$ is at most $M_{i}$.

By the inductive hypothesis, the edges $(x, \hat{x})$ and $(\hat{y}, y)$ whose weight is at most $2 \beta M_{i+1}$ are approximated with $1+\epsilon$ stretch. Thus, there is a path in the spanner from $x$ to $y$ whose length is at most $(1+\epsilon) \delta(x, \hat{x})+M_{i}+(1+\epsilon) \delta(\hat{y}, y)$, and this can be bounded by

$$
(1+\epsilon) 4 \beta M_{i+1}+M_{i}=((1+\epsilon) 4 \beta+(1+\alpha)) M_{i+1} .
$$

As the edge $(x, y) \in E\left(M_{i+1}, M_{i}\right)$ it follows that $\delta(x, y) \geq M_{i+1}$. It remains to prove that $1+4 \epsilon \beta+4 \beta+\alpha \leq 1+\epsilon$, which follows directly from the choice of $\alpha$ and $\beta$.

If $r(y)<M_{i+1}$ then there must be an edge $(\hat{x}, y) \in E_{\mathrm{SP}}^{\mathrm{DIR}}$, where $\hat{x} \in \Gamma_{i}(p)$. Following similar arguments to those used above it can be shown that there is a path in the spanner from $x$ to $y$ of length at most $(1+\epsilon) 2 \beta M_{i+1}+M_{i}$ and hence bounded by $(1+\epsilon) M_{i+1}$.

The size of the spanner. We now prove that the size of the spanner $H^{\mathrm{DIR}}\left(V, E_{\mathrm{SP}}^{\mathrm{DIR}}\right)$ is $O\left(n / \epsilon^{d} \log M\right)$. As a first step, we state the following well-known lemma, cf. [2].

Lemma 2.2. [Packing Lemma] If all points in a set $U \in \mathbb{R}^{d}$ are at least $r$ apart from each other, then there are at most $(2 R / r+1)^{d}$ points in $U$ within any ball $X$ of radius $R$.

The next lemma establishes a bound on the number of incoming spanner edges that a point may be assigned on stage $i \in\left[0,\left\lfloor\log _{1+\alpha} M\right\rfloor\right]$ of the algorithm.

Lemma 2.3. Let $i \in\left[0,\left\lfloor\log _{1+\alpha} M\right\rfloor\right]$ and let $y \in V$. The total number of incoming edges of $y$ that were added to the spanner on stage $i$ is $O\left(\epsilon^{-d}\right)$.

Proof. Let $(x, y)$ be a spanner edge and let $\mathrm{NN}\left(x, P_{i}\right)=p$. We associate $(x, y)$ to $p$. From the spanner construction algorithm it follows that this is the only incoming edge of $y$ whose source is in $\Gamma_{i}(p)$. Thus, this is the only incoming edge of $y$ which is associated to $p$. Now consider all the incoming edges of $y$ on stage $i$. The source of each of these edges is associated to a unique pivot within distance of at most $M_{i}+2 \beta M_{i+1}$ away from $y$ and any two pivots are $\beta M_{i+1}$ apart from each other. Using Lemma 2.2, we get that the number of edges entering $y$ is $\left(\frac{M_{i}+2 \beta M_{i+1}}{\beta M_{i+1}}+1\right)^{d}=((1+\alpha) / \beta+3)^{d}=O\left(\epsilon^{-d}\right)$.

It follows from the above lemma that the total number of edges that were added to $E_{\mathrm{SP}}^{\mathrm{DIR}}$ in the main loop is $O\left(n / \epsilon^{d} \log M\right)$. The total cost of the construction algorithm is $O(m \log n)$. For more details on the construction time see [8].

\subsection{Spanner for relaxed disk graphs}

Let $(V, \delta)$ be a metric space of constant doubling dimension $d$ with a radius assignment $r(\cdot)$ for its points and let $I=(V, E, r)$ be its corresponding disk graph. Assume that we multiply the radius assignment of every point by a factor of $1+\epsilon$, for some $\epsilon>0$, and let $I^{\prime}=\left(V, E^{\prime}, r_{1+\epsilon}\right)$ be the corresponding disk graph. In this section we show that it is possible to create a $(1+\epsilon)$-spanner of $I$ of size $O\left(n / \epsilon^{d}\right)$ if we are allowed to use edges of $I^{\prime}$.

Our construction consists of two stages: a building stage and a pruning stage. The building stage creates two spanners, $H$ and $H^{\prime}$, using the algorithm of Section 2.1, where $H$ is the spanner of $I$ and $H^{\prime}$ is the spanner of $I^{\prime}$. In the pruning stage we prune the union of these two spanners. Throughout the pruning stage we use the radius assignment of each 
point before the increase. Let $q \in V$ and let $\ell(q)$ be the first level in which $q$ can have outgoing edges, that is, $r(q) \in\left[M_{\ell(q)+1}, M_{\ell(q)}\right]$ (recall that as the levels get larger the edges get shorter). In the pruning stage we only prune incoming edges of $q$ whose level is below $\ell(q)$. In other words, we do not touch the incoming edges of $q$ that are shorter than the radius of $q$. The pruning is done as follow. Let $\gamma=\log _{1+\alpha} 1 / \beta+1$. We keep in the spanner the incoming edges of $q$ that come from the first $4 \gamma$ different levels below $\ell(q)$.

Let $\hat{H}$ be the resulting spanner and let $\hat{E}$ be the remaining set of edges after the pruning step. In the remainder of this section we show that the size of $\hat{H}$ is $O\left(n / \epsilon^{d}\right)$ and its stretch with respect to the distances in $I(V, E, r)$ is $1+\epsilon$. We start by showing that the size of $\hat{H}$ is $O\left(n / \epsilon^{d}\right)$. Notice that the first part of the proof below is possible only due to the change we have done in the previous section to our spanner construction from [8]. Roughly speaking, given an edge $(p, q) \in E$ that is shorter than $r(q)$ we use pivot selection also on $q$ 's side (and not only on $p$ 's) to sparisify the graph. This allows us to deal separately with edges of $q$ of length larger than $r(q)$ and those of length smaller than $r(q)$.

Lemma 2.4. $|\hat{E}|=O\left(n / \epsilon^{d}\right)$.

Proof. Let $(p, q)$ be a spanner edge that survived the pruning step. There are two possible cases to consider.

The first case is that $\ell(p, q)>\ell(q)$. Let $i=\ell(p, q)$ and let $x=\mathrm{NN}\left(p, P_{i}\right)$ and $y=$ $\mathrm{NN}\left(q, P_{i}\right)$. By packing considerations similar to Lemma 2.3 it follows that the total number of edges at level $i$ that connects between two pivots as the edge $(p, q)$ that are associated with $x$ (and with $y$ ) is $O\left(1 / \epsilon^{d}\right)$. The distance between $x$ and $y$ is at most $2 \beta M_{i+1}+M_{i}$, therefore at level $i-2 \gamma$ either $x$ or $y$ are no longer pivots.

Let $x \in P_{j}$ and $x \notin P_{j-1}$, that is, $P_{j}$ is the first pivot set that contains $x$. Then we charge $x$ with every (incoming and outgoing) edge of this type from levels $[j, j+2 \gamma]$ that is incident to $x$. Now given such an edge $(p, q)$ whose level is $i$, either $x$ or $y$ are not pivots in level $i-2 \gamma$, which means that either $x$ or $y$ has been charged for this edge, since one of them first becomes a pivot between levels $i-2 \gamma$ and $i$.

The second case is that $\ell(p, q) \leq \ell(q)$. In this case, it must be that level $\ell(p, q)$ is among the $4 \gamma$ first different levels below $\ell(q)$ from which an incoming edge is allowed to enter $q$. Subsequently, we associate the edge $(p, q)$ with $q$, as the total number of such edges that $q$ can have is $O\left(\gamma / \epsilon^{d}\right)$.

We now turn to prove that the stretch of the spanner $\hat{H}$ with respect to the disk graph $I$ is $1+\epsilon$.

Lemma 2.5. Let $(p, q)$ be an edge of the spanner $H$ that was pruned. We show that there is a path in $\hat{H}$ whose length is at most $(1+\epsilon) \delta(p, q)$.

Proof. The proof is by induction on the lengths of the pruned edges. For the induction base let $(p, q)$ be the shortest edge that was pruned. For every $x \in V$, let $s(x)$ be the head of an edge whose level is the $\gamma$-th level below $\ell(x)$ from which $x$ has an incoming edge. Let $q_{1}, \ldots q_{i}, \ldots$ be a sequence of points, where $q_{1}=q$ and $q_{i}=s\left(q_{i-1}\right)$. As $q_{i+1}=s\left(q_{i}\right)$, it follows that $\ell\left(q_{i+1}, q_{i}\right) \leq \ell\left(q_{i}\right)-\gamma$. Combining this with the fact that $\ell\left(q_{i}\right) \leq \ell\left(q_{i}, q_{i-1}\right)$ we get that $\ell\left(q_{i+1}, q_{i}\right) \leq \ell\left(q_{i}, q_{i-1}\right)-\gamma$. Therefore, $\delta\left(q_{i}, q_{i-1}\right) \leq \beta \delta\left(q_{i+1}, q_{i}\right)$.

The analysis distinguishes between two cases.

Case a: There is a point $q_{t}$ such that $\delta\left(q_{t}, q\right)>\beta \delta(p, q)$. This situation is depicted in Figure 3. (If there is more than one point that satisfies this requirement, take the one whose index is minimal.) 


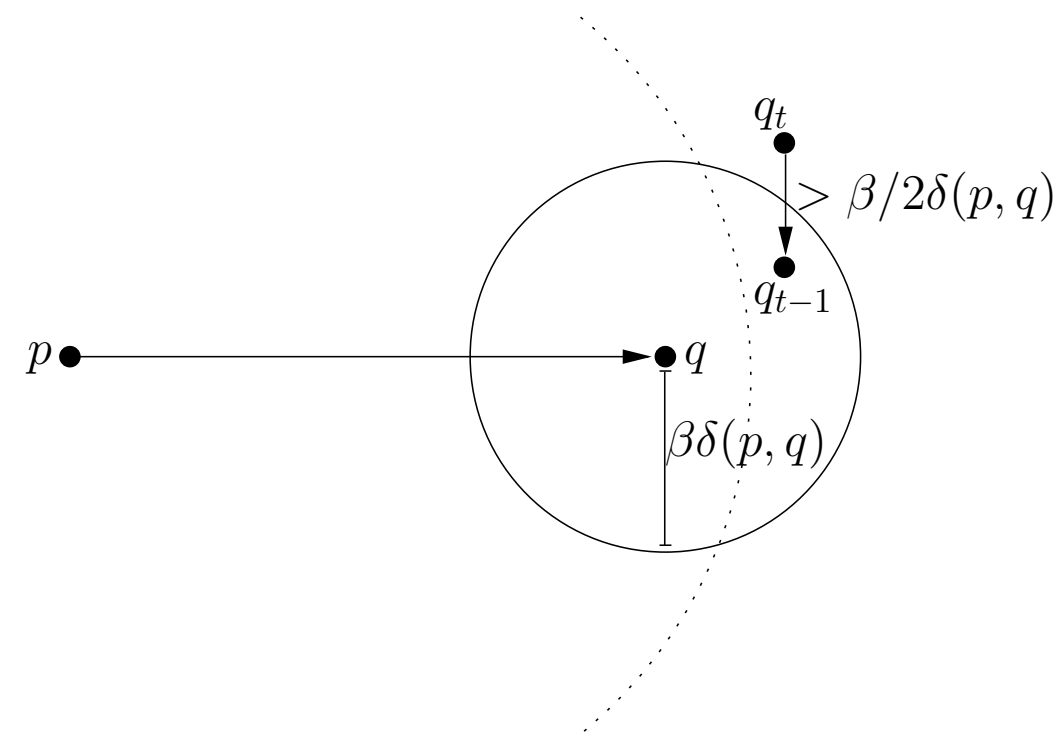

Figure 3: The case in which $q_{t}$ exists

Claim: $\delta\left(q_{t}, q_{t-1}\right) \geq \frac{\beta}{2} \delta(p, q)$.

Proof. For the sake of contradiction, assume that $\delta\left(q_{t}, q_{t-1}\right)<\frac{\beta}{2} \delta(p, q)$. This implies that

$$
2 \delta\left(q_{t}, q_{t-1}\right)<\beta \delta(p, q)<\delta\left(q_{t}, q\right) \leq \sum_{i=2}^{t} \delta\left(q_{i}, q_{i-1}\right)
$$

where the last inequality follows from the triangle inequality as the distance between $q$ and $q_{t}$ is at most $\sum_{i=2}^{t} \delta\left(q_{i-1}, q_{i}\right)$. For every $2 \leq i \leq t-1$ we have $\delta\left(q_{i}, q_{i-1}\right) \leq \beta \delta\left(q_{i+1}, q_{i}\right)$, which implies that $\delta\left(q_{i}, q_{i-1}\right) \leq \beta^{t-i} \delta\left(q_{t}, q_{t-1}\right)$. Combined with $(2.1)$, we get

$$
\delta\left(q_{t}, q_{t-1}\right)<\sum_{i=2}^{t-1} \delta\left(q_{i}, q_{i-1}\right) \leq \delta\left(q_{t}, q_{t-1}\right) \sum_{i=2}^{t-1} \beta^{t-i} .
$$

If $\beta<1 / 2$ we have $\sum_{i=2}^{t-1} \beta^{t-i}<1$ and this yields a contradiction.

We now focus our attention on the point $q_{t-1}$. The minimality of $q_{t}$ implies that $\delta\left(q, q_{t-1}\right) \leq \beta \delta(p, q)$. By combining it with the triangle inequality we get that $\delta\left(p, q_{t-1}\right) \leq$ $\delta(p, q)+\beta \delta(p, q)$. Therefore, in the graph $I^{\prime}$ there must be an edge from $p$ to $q_{t-1}$.

Let $i=\ell\left(p, q_{t-1}\right)$. There are two possible scenarios for the spanner $H^{\prime}$. The first scenario is when $r^{\prime}\left(q_{t-1}\right)<M_{i+1}$. In this case, there is an edge in $H^{\prime}$ from some $x \in \Gamma_{i}(\mathrm{NN}(p, i))$ to $q_{t-1}$, whose length is at most $\delta(p, q)+\beta \delta(p, q)$.

There are $4 \gamma$ different levels below $\ell\left(q_{t-1}\right)$ from which edges that belong to the spanners $H$ and $H^{\prime}$ are not being pruned and survived to the spanner $\hat{H}$. We know that the edge $\left(q_{t}, q_{t-1}\right)$ is such an edge from the $\gamma$-th non-empty level below $\ell\left(q_{t-1}\right)$. We also know that $\delta\left(q_{t}, q_{t-1}\right)>\frac{\beta}{2} \delta(p, q)$. Therefore, as the length of the edge $\left(x, q_{t-1}\right)$ is at most $\delta(p, q)+$ $\beta \delta(p, q)$ it is within the $4 \gamma$ non-empty levels below $\ell\left(q_{t-1}\right)$ and it is not pruned. We can now build a path from $p$ to $q$ by concatenating three segments as follows: A path from $p$ to $x$, the edge $\left(x, q_{t-1}\right)$ and a path from $q_{t-1}$ to $q$. The point $x$ is at most $2 \beta \delta(p, q)+2 \beta^{2} \delta(p, q)$ 
away from $p$ and for the right choice of $\beta$ it is less than $\delta(p, q) /(1+\epsilon)$, hence the weight of every edge on the path that approximates the distance between $x$ and $p$ in $H \cup H^{\prime}$ is less than $\delta(p, q)$, the shortest pruned edge, and the entire path survived the punning stage. Similarly, the point $q_{t-1}$ is at most $\beta \delta(p, q)$ away from $q$ and again for the right choice of $\beta$ every edge on the path that approximates the distance between $q_{t-1}$ and $q$ survived the punning stage. Thus, we get that there is a path whose length is at most

$$
(1+\epsilon)\left(3 \beta \delta(p, q)+2 \beta^{2} \delta(p, q)\right)+\delta(p, q)+\beta \delta(p, q),
$$

which is less than $(1+\epsilon) \delta(p, q)$ for $\beta<\epsilon / 11$.

The second scenario is when $r^{\prime}\left(q_{t-1}\right) \geq M_{i+1}$. In this case, there is an edge in $H^{\prime}$ from some $x \in \Gamma_{i}(\mathrm{NN}(p, i))$ to some $y \in \Gamma_{i}\left(\mathrm{NN}\left(q_{t-1}, i\right)\right)$ whose length is at most $\delta(p, q)+\beta \delta(p, q)$, which is not being pruned. We can build a path from $p$ to $q$ by concatenating three segments as follows: A path from $p$ to $x$, the edge $(x, y)$ and a path from $y$ to $q$. As before, for the right choice of $\beta$ the paths from $p$ to $x$ and from $y$ to $q$ are composed from edges that are shorter from $\delta(p, q)$, the length of the shortest pruned edge, hence, from the minimality $\delta(p, q)$ every edge on these paths survived the punning stage. We get that there is a path whose length is at most

$$
(1+\epsilon)\left(4 \beta \delta(p, q)+5 \beta^{2} \delta(p, q)\right)+\delta(p, q)+\beta \delta(p, q)
$$

which is less than $(1+\epsilon) \delta(p, q)$ for $\beta<\epsilon / 19$. This completes the proof for case a.

Case b: There is no point $q_{t}$ such that $\delta\left(q_{t}, q\right)>\beta \delta(p, q)$. In this case, let $q_{t-1}$ be the last point in the sequence of points $q_{1}, \ldots q_{i}, \ldots$, where $q_{i}=s\left(q_{i-1}\right)$ and $q_{1}=q$. Similarly to before, there are two possible scenarios for the spanner $H^{\prime}$. Let $i=\ell\left(p, q_{t-1}\right)$. The first scenario is when $r^{\prime}\left(q_{t-1}\right)<M_{i+1}$. In this case, there is an edge in $H^{\prime}$ from some $x \in \Gamma_{i}(\mathrm{NN}(p, i))$ to $q_{t-1}$ whose length is at most $\delta(p, q)+\beta \delta(p, q)$. This edge could not be pruned, since if it was pruned then $q_{t-1}$ could not have been the last point in the sequence. Hence we can construct a path from $p$ to $q$ exactly as we have done in the first scenario of case a, described above. The second scenario is when $r^{\prime}\left(q_{t-1}\right) \geq M_{i+1}$. In this case, we can construct a path from $p$ to $q$ exactly as we have done in the second scenario of case a, described above.

This completes the proof of the induction base. The proof of the general inductive step is almost identical. The only difference is that when a path is constructed from $p$ to $q$, its portions from $p$ to $x$ and from $q_{t-1}$ to $q$ in the first scenario and from $p$ to $x$ and from $y$ to $q$ in the second scenario exist in $\hat{H}$ by the induction hypothesis and not by the minimality of $\delta(p, q)$.

We end this section by stating its main Theorem. The proof of this Theorem stems from Lemma 2.4 and Lemma 2.5.

Theorem 2.6. Let $(V, \delta)$ be a metric space of constant doubling dimension with a radius assignment $r(\cdot)$ for its points and let $I=(V, E, r)$ be its corresponding disk graph. Let $I^{\prime}=\left(V, E^{\prime}, r_{1+\epsilon}\right)$ be the corresponding disk graph in the relaxed model. It is possible to create $a(1+\epsilon)$-spanner of size $O\left(n / \epsilon^{d}\right)$ for $I$ using edges of $I^{\prime}$.

\section{Concluding remarks and open problems}

This paper presents a spanner construction for disk graphs in a slightly relaxed model that is as good as spanners for complete graphs and unit disk graphs. This result opens 
many other research directions for disk graphs. We list here two questions that we find particularly intriguing: Is it possible to design an efficient compact routing scheme for disk graphs? And is it possible to build an efficient distance oracle for disk graphs?

\section{References}

[1] U. Zwick D. Dor, S. Halperin. All-pairs almost shortest paths. SIAM J. Comput., 29(5):1740-1759, 2000.

[2] J. Gao, L. Guibas, and A. Nguyen. Deformable spanners and applications. In Proc. 20th ACM Symp. on Computational Geometry, pages 179-199, 2004.

[3] Jie Gao, Leonidas J. Guibas, John Hershberger, Li Zhang, and An Zhu. Geometric spanners for routing in mobile networks. IEEE J. on Selected Areas in Communications, 23(1):174-185, 2005.

[4] Sariel Har-Peled and Manor Mendel. Fast construction of nets in low-dimensional metrics and their applications. SIAM J. Computing, 35:1148-1184, 2006.

[5] C. Wagner L. Cowen. Compact roundtrip routing for digraphs. In SODA '99: Proceedings of the tenth annual ACM-SIAM symposium on Discrete algorithms, pages 885-886, Philadelphia, PA, USA, 1999. Society for Industrial and Applied Mathematics.

[6] U. Zwick L. Roditty, M. Thorup. Roundtrip spanners and roundtrip routing in directed graphs. $A C M$ Trans. Algorithms, 4(3):1-17, 2008.

[7] Xiang-Yang Li, Gruia Calinescu, Peng-Jun Wan, and Yu Wang. Localized delaunay triangulation with application in ad hoc wireless networks. IEEE Trans. on Parallel and Distributed Systems, 14(10):10351047, 2003.

[8] D. Peleg and L. Roditty. Localized spanner construction for ad hoc networks with variable transmission range. In Proc. 7th Int. Conf. on Ad-Hoc Networks and Wireless (AdHoc-NOW), pages 622-633, 2008.

[9] L. Roditty. Fully dynamic geometric spanners. symposium on computational geometry. pages 373-380, 2007.

[10] Yu Wang and Xiang-Yang Li. Efficient delaunay-based localized routing for wireless sensor networks. Int. J. of Communication Systems, 20(7):767-789, 2006.

[11] Andrew Chi-Chih Yao. On constructing minimum spanning trees in k-dimensional spaces and related problems. SIAM J. Comput., 11(4):721-736, 1982. 